\title{
All-Organic Actuator Fabricated with Single Wall Carbon
}

\author{
Nanotube Electrodes
}

\author{
Jin Ho Kang, Cheol Park
}

National Institute of Aerospace, Hampton, VA 23666

Sharon E. Lowther, Joycelyn S. Harrison

Advanced Materials and Processing Branch, NASA Langley Research Center, Hampton, VA 23681

\section{Chan Eon Park}

Polymer Research Institute, Department of Chemical Engineering, Pohang University of Science and Technology (POSTECH), Pohang, 790-784, Korea

\begin{abstract}
Compliant electrodes to replace conventional metal electrodes have been required for many actuators to relieve the constraint on the electroactive layer. Many conducting polymers have been proposed for the alternative electrodes, but they still have a problem of poor thermal stability. This article reports a novel all-organic actuator with single wall carbon nanotube (SWCNT) films as the alternative electrode. The SWCNT film was obtained by filtering a SWCNT solution through an anodized alumina membrane. The conductivity of the SWCNT film was about $280 \mathrm{~S} / \mathrm{cm}$. The performance of the SWCNT film electrode was characterized by measuring the dielectric properties of NASA Langley Research Center - Electroactive Polymer (LaRC-EAP) sandwiched by the SWCNT electrodes over a broad range of temperature (from $25^{\circ} \mathrm{C}$ to $280^{\circ} \mathrm{C}$ ) and frequency (from $1 \mathrm{KHz}$ to $1 \mathrm{MHz}$ ). The all-organic actuator with the SWCNT electrodes
\end{abstract}


showed a larger electric field-induced strain than that with metal electrodes, under identical measurement conditions.

Keywords: actuator; single wall carbon nanotube; electrodes; high performance polymer; nanocomposite; polyimide

\section{INTRODUCTION}

Electroactive materials have been used in a number of applications that require electromechanical energy conversion, including electromechanical sensors and actuators, transducers, sonars, medical devices, prosthetics, artificial muscles, and materials for vibration and noise control. ${ }^{1}$ Electroactive polymers possess many advantages over piezoceramic and shape memory alloys in these applications, owing to their light weight, conformability, high toughness, and tailorable properties. In addition, electroactive polymers have high voltage sensitivity and low acoustic impedance, which enables medical and underwater applications. ${ }^{1,2}$

Recently, a high performance electroactive polyimide nanocomposite was developed by adding single wall carbon nanotubes (SWCNTs) into NASA Langley Research Center - Electroactive Polymer (LaRC-EAP). ${ }^{3}$ The addition of SWCNTs led to significant enhancement of the electrostrictive strain due to the increase of interfacial polarization. This electroactive polyimide nanocomposite (SWCNT/LaRC-EAP nanocomposite) exhibits superb actuation properties and can withstand high temperatures with improved mechanical integrity and chemical stability. ${ }^{3}$ 
As interest in high performance polymer based electroactive devices increases, the need for new electrode materials has emerged. Typical polymeric electroactive devices use metal electrodes, such as silver or gold, to provide electric fields. These metal electrodes often inhibit the displacement (elongation or contraction) of the electroactive polymer layer because they have a much smaller compliance (greater modulus) than the active polymer. Thus, the actual electric field-induced strain output of the devices with metal electrodes is always smaller than what they could intrinsically generate. $^{4,5}$

Conducting polymers have been used as alternative electrodes for electroactive polymeric devices. ${ }^{6-8}$ These electrodes effectively relieved the restraint on the movement in the active layer because their compliance is similar to that of the active polymer layer. The resulting devices exhibited higher strain than those with metal electrodes. However, the conducting polymers have a disadvantage of low conductivity at high temperatures because of dehydration and/or dedoping phenomena, and can not be used in applications that require high thermal stability. Therefore, the need still exists for an alternative electrode with higher compliance than the conventional metallic electrodes and with good thermal stability.

Recently, a novel method of forming optically transparent and electrically conductive SWCNT films was reported. ${ }^{9,10}$ This method consists of dispersing SWCNTs into a solution, filtering the SWCNT solution onto the surface of a porous membrane to form a SWCNT film, and then separating the SWCNT film from the porous membrane by dissolving the membrane. The SWCNT film showed a sheet resistance of less than $200 \Omega / \square$ and at least $30 \%$ transmission at a wavelength of $3 \mu \mathrm{m}$. The SWCNT film has a 
porous mat structure, and a high elastic compliance. The high elastic compliance seems to help reduce the constraint on the electroactive polymeric layer. Pasquier et al. reported on a polymer solar cell fabricated with a SWCNT transparent film as an electrode,,${ }^{11}$ but there is no report of an actuator device fabricated with a SCWNT film as an electrode.

This article describes the development of a novel all-organic actuator fabricated using SWCNTs as electrodes. Its electrical and electromechanical characteristics are reported and compared to those to metal electrodes.

\section{EXPERIMENTAL}

\section{Materials}

Purified HiPco (high pressure CO) SWCNTs were purchased from CNI (Carbon Nanotechnologies, Inc.). The polyimide (LaRC-EAP) matrix was synthesized with a diamine, 2,6-bis(3-aminophenoxy) benzonitrile (( $\beta$-CN)APB), and a dianhydride, 4,4oxydiphthalic anhydride (ODPA). The SWCNT/LaRC-EAP nanocomposite film as an actuation layer was synthesized by in-situ polymerization under simultaneous sonication

and shear. ${ }^{12}$ The synthesis scheme is shown in Figure 1 . The concentration of the SWCNTs in the polyimide was $0.1 \mathrm{wt} \%$. The complete synthetic procedure is described in detail elsewhere. ${ }^{12}$

The SWCNT electrode film was prepared using a method similar to the one reported in reference 9. The HiPco SWCNTs were dispersed into N,NDimethylacetamide (DMAc) at a concentration of $0.09 \mathrm{wt} \%$ under sonication for $2 \mathrm{~min}$ at $40 \mathrm{~W}$ and $20 \mathrm{kHz}$ (Misonix Sonicator ${ }^{\circledR} 3000$ ) and subsequently for $30 \mathrm{~min}$ at $80 \mathrm{~W}$ and 40 
$\mathrm{kHz}$ (Fisher Scientific, FS 20), then filtered onto the surface of an anodized alumina membrane (pore size: $0.2 \mu \mathrm{m}$ ) to form a SWCNT film on the membrane. An example of a filtered SWCNT film on the membrane is shown in Figure 2(a). A freestanding SWCNT film was easily released from the substrate by breaking the brittle membrane as seen in Figure 2(b).

A conventional metal electroded actuator (metal electrodel actuation layer (SWCNT/LaRC-EAP nanocomposite) metal electrode) was fabricated by sputtering silver (about $90 \mathrm{~nm}$-thick) onto the SWCNT/LaRC-EAP nanocomposite film (actuation layer). The all-organic actuator (SWCNT electrodel actuation layer (SWCNT/LaRC-EAP nanocomposite) (SWCNT electrode) was fabricated by pressing the SWCNT/LaRC-EAP nanocomposite film with the SWCNT electrode film layers on both sides at a predetermined pressure. Three different samples were prepared by pressing under a pressure of 600,3000 and $6000 \mathrm{psi}$, respectively. A schematic of the cross-sectional view of the layers of the all-organic actuator is shown in Figure 3(a). The pressing temperature and time were $230^{\circ} \mathrm{C}$ and $2 \mathrm{~min}$, respectively. All the samples were pre-heated at $230^{\circ} \mathrm{C}$ for $20 \mathrm{~min}$ prior to pressing. Silicone elastomer films were used as extra layers to cover the actuator so as to apply uniform stress (Figure 3(a)). Figure 3(b) shows an example of the all-organic actuator film pressed with the SWCNT electrodes. When the SWCNT electrode was sub-micron thick, the all-organic actuator film was transparent as shown in Figure 3 (b). However, due to the difficulty in handling such a thin film, the SWCNT electrode film used in the paper was $2-3 \mu \mathrm{m}$ thick.

\section{Characterization}


Cross-sectional images of the actuator were obtained by a High Resolution Scanning Electron Microscope (HRSEM) (Hitachi S-5200) after sectioning the actuator with a microtome (Leica Ultracut UCT) down to approximately $70 \mathrm{~nm}$ thick.

The dielectric properties and AC conductivities of the SWCNT/LaRC-EAP nanocomposites layered with either metal electrodes or SWCNT electrodes were measured as a function of frequency and temperature using a Novocontrol-Solartron 1260 impedance/gain-phase analyzer. DC conductivities were measured by a 4-point probe measurement system (Signatone, QuadPro Resistivity Wafer Mapping System).

To investigate the actuation characteristics, out-of-plane displacement measurements were performed using a non-contacting fiber optic sensor manufactured by Opto-Acoustic Sensors (Figure 4). Sinusoidal waveforms at a frequency of $1 \mathrm{~Hz}$ were used for all measurements. Multiple samples (at least 3 samples) were measured at each condition, and the average displacement was recorded. Out-of-plane polymer motion was determined by monitoring the intensity of the light reflected from the actuator surface. As the surface of the polymer film moves away from the sensor tip, the signal decreases, and as the surface moves closer to the sensor tip, the signal increases. The out-of-plane strain $\left(S_{33}\right)$ was measured in response to the applied electric field $(E)$, which is given as,

$$
S_{33}=\Delta t / t
$$

where $t$ is the thickness of the polymer and $\Delta t$ is the change in thickness.

\section{RESULTS AND DISCUSSION}




\section{Mophologies of the All-Organic Actuator}

A free standing 2-3 $\mu \mathrm{m}$ thick SWCNT electrode film having a DC conductivity of 280 S/cm was obtained. The all organic-actuator layered with these SWCNT electrodes was fabricated using a hydraulic press as described in the experimental section. Figures 5(a) and (b) show cross-sectional HRSEM images of the SWCNT electrodes after pressed at 600 and $6000 \mathrm{psi}$, respectively. While the cross-sectional structure of the SWCNT electrode pressed onto the actuation layer under 600 psi showed a slightly porous morphology (Figure 5(a)), the one prepared under 6000 psi had a smooth and compact morphology without any noticeable porosity (Figure 5(b)). It is anticipated that denser SWCNT electrodes have higher elastic moduli to constrain the movement of their actuation layer.

\section{Dielectric and Electrical Properties at Elevated Temperatures}

Although conducting polymers have been used as alternative electrodes for electroactive polymeric devices, most research on conducting polymers have shown that their thermal stability limits the range of their use to less than about $120^{\circ} \mathrm{C}$. This is because the conducting polymers lose their conductivities at elevated temperatures due to dedoping effects often caused by dehydration., ${ }^{4,6-8}$ For aerospace and other "harsh" environment applications, actuators should survive at a temperatures of at least $150^{\circ} \mathrm{C}$ or higher. ${ }^{13}$ Therefore, it is necessary to examine whether the SWCNT electrodes can operate properly over a broad range of temperatures. The temperature and frequency dependence of the dielectric constant (real part) for the SWCNT/LaRC-EAP nanocomposite layered 
with the SWCNT electrodes is shown in Figure 6(a). It shows a similar trend to that of the SWCNT/LaRC-EAP nanocomposite layered with gold electrodes up to the glass transition temperature (Figure 6(b)). The dielectric constant remained constant up to $210^{\circ} \mathrm{C}$, and then increased rapidly. The increase of dielectric constant above $210^{\circ} \mathrm{C}$ is due to the higher dipolar mobility of LaRC-EAP above the glass transition temperature $\left(T_{g}\right)$, which is around $210^{\circ} \mathrm{C}$. As frequency decreases, these dipoles might have more time to rotate themselves along the applied electric field at each cycle, resulting in a higher dielectric constant, which is observed for both SWCNT and metal electrode actuators. AC conductivities of SWCNT/LaRC-EAP nanocomposites layered with the SWCNT electrodes and the metal electrodes are shown in Figure 7 as a function of temperature. The overall trend of AC conductivities for both samples prepared with different electrodes was almost identical, especially at lower temperatures, indicating that the SWCNT electrode performs like the metal electrode. The AC conductivities increased with increasing temperature due to the increased mobility of charge carriers at higher temperature, which is typical for non-metallic materials. Both samples showed higher AC conductivity as frequency increased. It is a generally observed that the higher frequency gives rise to more polarization conductivity for non-metallic materials. ${ }^{14}$ Based on the consistent electrical and dielectric data, it is believed that the SWCNT electrodes are suitable replacements for gold electrodes in harsh conditions at elevated temperatures (at $210^{\circ} \mathrm{C}$ or above). In fact, pristine SWCNTs do not oxidize up to $400^{\circ} \mathrm{C}$ in air.

\section{Electroactive Response of All-Organic Actuator}


Figure 8 shows the electric field-induced strain for these two actuators, a SWCNT/LaRCEAP nanocomposite layered with the metal electrodes as a reference and three SCWNT/LARC-EAP nanocomposites layered with the SWCNT electrodes at three different pressures. The out-of-plane strain $\left(S_{33}\right)$ through the film thickness was plotted as a function of the applied electric field strength. The strain $\left(S_{33}\right)$ of all SWCNT/LaRCEAP nanocomposite actuators increased quadratically with increasing applied electric field, indicating that the strain mainly originated from an electrostrictive response. The effect of SWCNT on an electrostrictive response of the SWCNT/LaRC-EAP nanocomposite has been reported by Kang et al. ${ }^{3}$ The large interfaces between the highly conductive SWCNT inclusions and the insulating matrix of LaRC-EAP generate high interfacial polarization when space charges build up at the interfaces due to the mismatch of the conductivities and dielectric constants of two phases. This is called MaxwellWagner-Sillar interfacial polarization. ${ }^{15,16}$ This type of polarization principally influences the low frequency $\left(10^{-5}\right.$ to $\left.10^{2} \mathrm{~Hz}\right)$ dielectric properties significantly. As the frequency decreases, the time available for the drift of charge carriers increases and observed values of dielectric constant become significantly higher, as shown in Figure 6 . The conductivity term in interfacial polarization also makes an increasing contribution to the dielectric loss as the frequency becomes smaller. Our previous study of this nanocomposite showed the characteristic dielectric spectra for the interfacial polarization as well. ${ }^{3}$ This high interfacial polarization appears to be the origin of the high electroactive response under an electric field.

The electrostrictive coefficient $\left(M_{33}\right)$ of the SWCNT/LaRC-EAP nanocomposite with the silver electrodes was $1.58 \times 10^{-15} \mathrm{~m}^{2} / \mathrm{V}^{2}$, which was calculated from the slope of 
the plot of the strain $\left(S_{33}\right)$ to the square of electric field strength $(E), S_{33}=M_{33} E^{2}$. The SWCNT/LaRC-EAP nanocomposite layered with the SWCNT electrodes pressed at 6000 psi exhibited the same level of strain as that with the silver electrodes. With decreasing the fabrication pressure of the SWCNT electrodes, the strain increased as shown in Figure 8. The strain of the SWCNT/LaRC-EAP nanocomposite layered with the SWCNT electrodes pressed at $600 \mathrm{psi}$ increased much more rapidly than that with the silver electrodes as a function of the applied electric field. The electrostrictive coefficient $\left(M_{33}\right)$ of this SWCNT electroded actuator was $3.86 \times 10^{-15} \mathrm{~m}^{2} / \mathrm{V}^{2}$, which is more than two times higher than that of the silver electroded actuator. Thus, the all organic actuator shows over $240 \%$ increase in electroactive strain compared with the silver electroded actuator under identical applied electric field. This significant increase of strain indicates that the compliant SWCNT electrodes relieve the restraint of movement of the active layer. All of the SWCNT/LaRC-EAP nanocomposite with SWCNT electrodes showed higher strain than those with silver electrodes regardless of the employed fabrication pressure. Indirect calculations, using the four-layer model of the actuator (and based on the measured strain, the modulus of the SWNT/LaRC-EAP and the actuator dimension) indicate that the modulus of the SWCNT electrode itself ranges from 1 to $2 \mathrm{GPa}^{3,17}$ This is in the same range as that of the SWCNT/LaRC-EAP nanocomposite actuator layer (about 3GPa). The modulus or compliance (inverse modulus) of the SWCNT electrode can be tailored to match the actuation layer mechanical properties to maximize the actuation.

\section{CONCLUSIONS}


A SWCNT film was used as a compliant electrode to fabricate an "all-organic actuator" by layering the SWCNT films on the both sides of SWCNT/LaRC-EAP nanocomposite (actuating layer) film. The SWCNT film can relieve the constraint on the electroactive layer to generate higher electomechanical output. The dielectric and electrical properties of an actuator layered with the SWCNT electrodes were measured to compare with those constructed with conventional metal electrodes. The overall trends of both dielectric and electrical properties of both samples were very similar, indicating that the SWCNT film electrodes can be used as complaint electrodes at harsh conditions like metal electrodes. Moreover, the all-organic actuators with the SWCNT electrodes showed a larger electric field-induced strain $(240 \%)$ than that with metal electrodes under identical measurement conditions, since the SWCNT electrodes can relieve the restraint of movement of the active layer more readily. When a sub-micrometer SWCNT electrode film was used, a transparent all-organic actuator, which can be applicable to various optical devices was obtained. In summary, the SWCNT film can be used as a good compliant electrode for enhancing and tailoring the performance of actuators.

\section{ACKNOWLEDGEMENTS}

The authors thank Gregory K. Draughon and Nancy M. Holloway for their help in preparation of the electrode-deposited film. Park and Kang appreciate NASA University Research, Engineering and Technology Institute on Bio Inspired Materials (BIMat) under award no. NCC-1-02037 for support in part. Kang appreciates the Post-doctoral 
Fellowship Program of Korea Science and Engineering Foundation (KOSEF) under grant no. M01-2004-000-10344-0 for support in part. 


\section{References}

1. Uchino, K. Piezoelectric Actuators and Ultrasonic Motors; Kluwer Academic: Boston; 1997.

2. Ounaies, Z.; Young J. A.; Harrison, J. S. Field Responsive Polymers; Khan, I. M.; Harrison, J. S., Eds.; ACS: Washington DC, 1999; Chapter 6, pp. 88-103.

3. Park, C.; Kang, J. H.; Ounaies, Z.; Costen, R. C.; Lowther, S. E.; Harrison, J. S. Adv Mater, in press.

4. Xu, H.-S;. Cheng, Z.-Y.; Bharti, V.; Wang, S.; Zhang, Q. M. J Appl Polym Sci 2000, $75,945-951$.

5. Pelrine, R. E.; Kornbluh, R. D.; Joseph, J. P. Sensors and Actuators A 1998, 64, 7785.

6. Ennis, B. C.; Truong, V. T. Synth Met 1993, 59, 387-399.

7. Biswas, M.; Roy, A. J Appl Polym Sci 1996, 60, 143.

8. Truong, V. T.; Ennis, B. C.; Forsyth, M. Polymer 1995, 36, 1933-1940.

9. Rinzler, A. G.; Chen, Z. (University of Florida). U.S. Patent Application Publication, 20040197546, October 7, 2004.

10. Wu, Z.; Chen, Z.; Du, X.; Logan, J. M.; Sippel, J.; Nikolou, M.; Kamaras, K.;

Reynolds, J. R.; Tanner, D. B.; Hebard, A. F.; Rinzler, A. G. Science 2004, 305, 1273-1276.

11. Pasquier, A. D.; Unalan, H. E.; Kanwal, A.; Miller, S.; Chhowalla, M. Appl Phys Lett $2005,87,203511$. 
12. Park, C.; Ounaies, Z.; Watson, K.; Crooks, R. E.; Smith Jr., J.; Lowther, S.E.; Connell, J. W.; Siochi, E. J.; Harrison, J. S.; St. Clair, T. L. Chem Phys Lett 2002, 364, 303308.

13. http://www.nasa.gov/mission_pages/exploration/mmb/index.html, http://www.hq.nasa.gov/osf/faq.htm\#17, http://www.centennialofflight.gov/essay/Evolution_of_Technology/TPS/Tech41.htm

14. Elimat, Z. M. J Phys D Appl Phys 2006, 39, 2824-2828.

15. Ku, C. C.; Liepins, R. Electrical Properties of Polymers; Hanser Publishers: New York, 1987; pp. 20 - 58.

16. Sillars, R. W. J Inst Electron Engrs 1937, 80, 378-394.

17. Singer, F. L. Strength of Materials; Harper \& Brothers: New York, 1951; pp 124-127. 


\section{Figure captions}

Figure 1. Schematic diagram of synthesis of SWCNT/LaRC-EAP (SWCNT/polyimide) nanocomposite.

Figure 2. (a) Filtered SWCNT film on anodized alumina membrane (pore size: $0.2 \mu \mathrm{m}$ ) and (b) the freestanding SWNT film delaminated by breaking the membrane.

Figure 3. (a) Schematic diagram of preparing an all-organic actuator (cross-section view) and (b) a prototype actuator film.

Figure 4. Schematic diagram of apparatus used to measure electromechanical actuation.

Figure 5. The cross-sectional SEM images of SWNT electrode (a) after pressing at 600 psi and (b) after pressing at 6000 psi.

Figure 6. The dielectric constant of (a) SWCNT/LaRC-EAP nanocomposite with SWNT electrodes and (b) SWCNT/LaRC-EAP nanocomposite with metal electrodes as a function of temperature and frequency.

Figure 7. AC conductivities of SWCNT/LaRC-EAP nanocomposite layered with SWNT electrodes $(\circ)$ and layered with metal electrodes $(\triangle \nabla)$ as a function of temperature and frequency.

Figure 8. The electric field-induced strain of SWCNT/LaRC-EAP nanocomposite layered with metal electrodes $(\square)$ and with SWNT electrodes $(\triangle \triangle \nabla)$. 


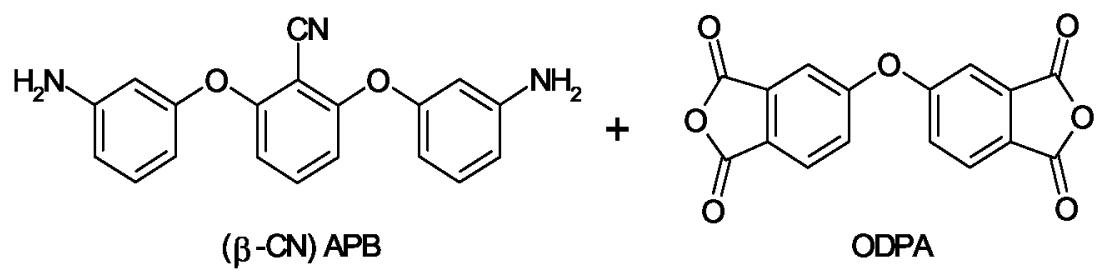

RT, $\mathrm{N}_{2}, \mathrm{DMF}$

SWNTs, under sonication
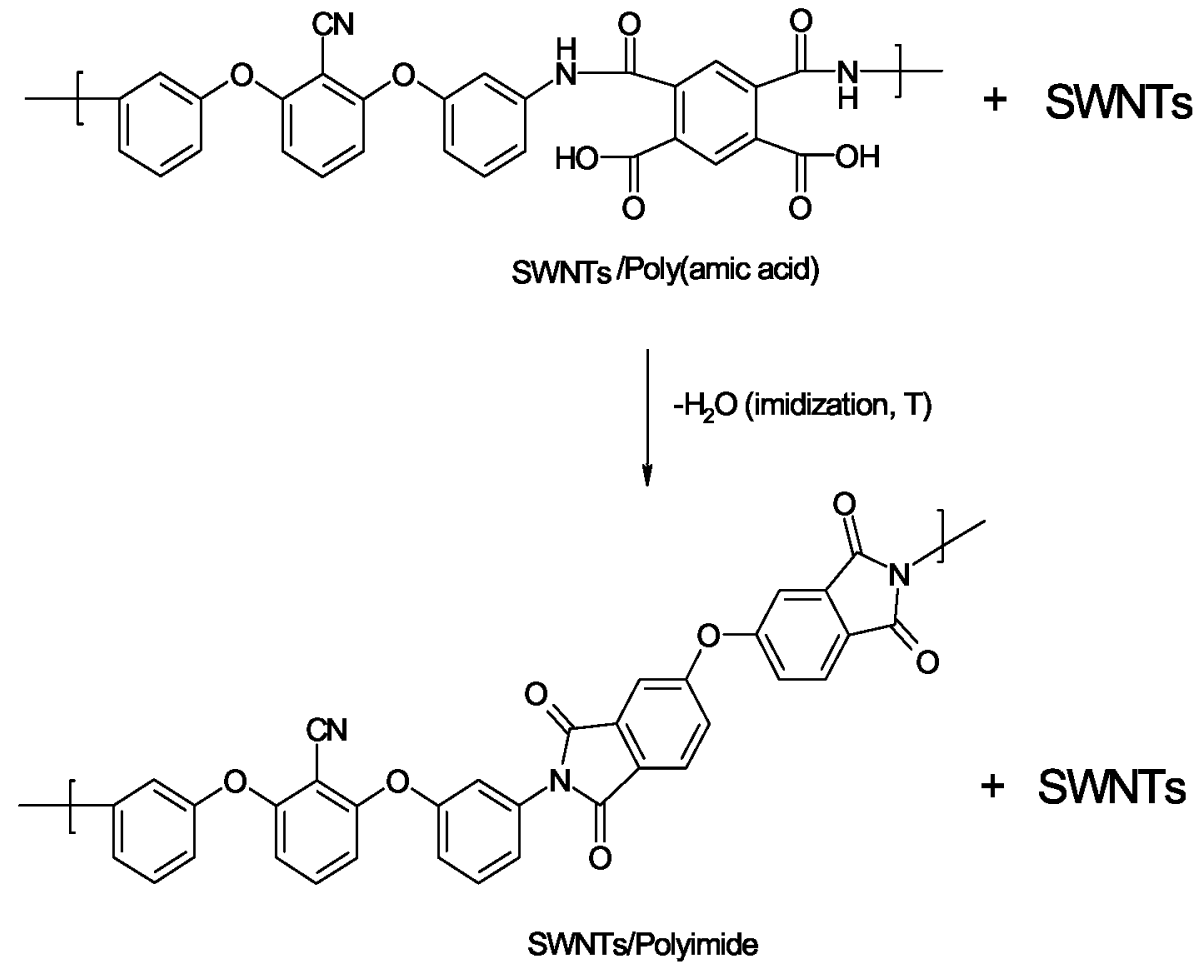

Figure 1. Schematic diagram of synthesis of SWCNT/LaRC-EAP (SWCNT/polyimide) nanocomposite.

Kang, Park, Lowther, Harrison, and Park 
(a)

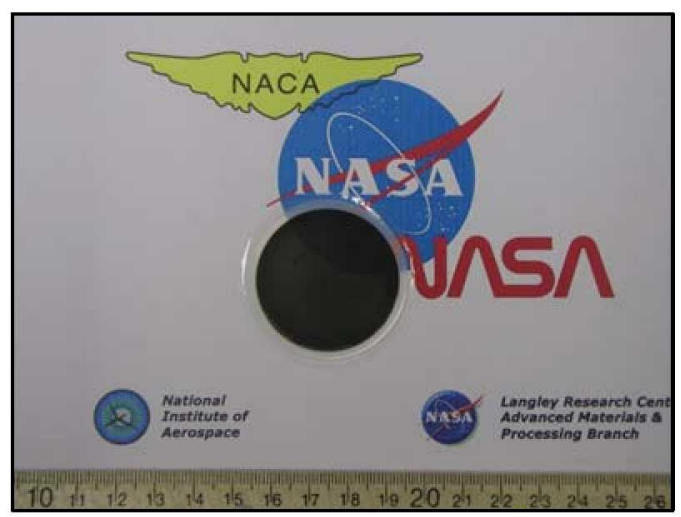

(b)

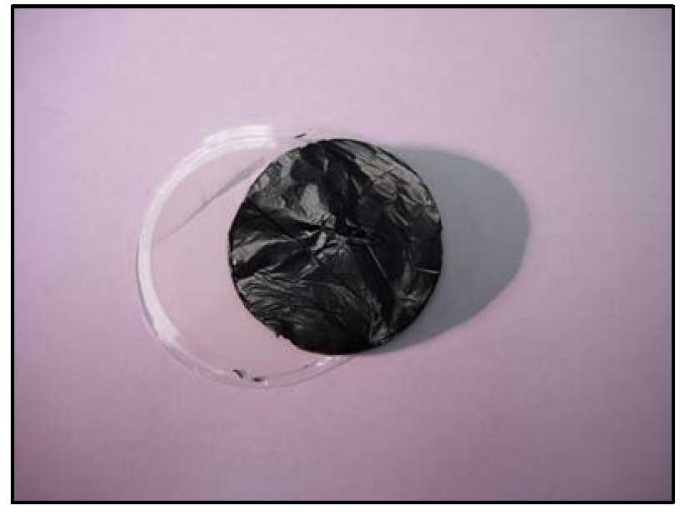

Figure 2. (a) Filtered SWCNT film on anodized alumina membrane (pore size: $0.2 \mu \mathrm{m}$ ) and (b) the freestanding SWNT film delaminated by breaking the membrane.

Kang, Park, Lowther, Harrison, and Park 
(a)

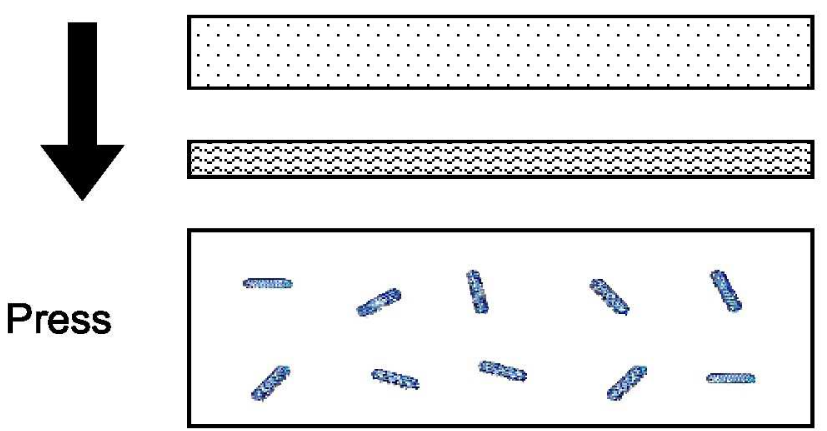

$\uparrow$
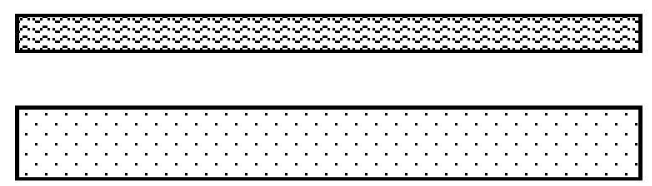

(b)

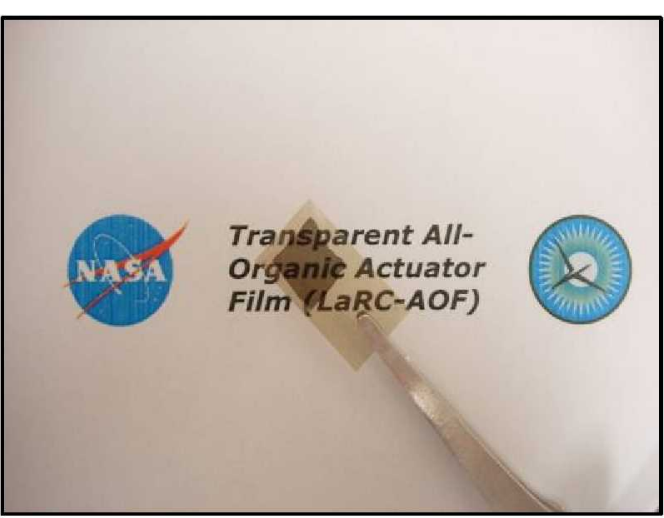

Silicone Elastomer

SWNT Electrode

SWCNT/LaRC-EAP

Nanocomposite

Actuating Laver

SWNT Electrode

Silicone Elastomer

Figure 3. (a) Schematic diagram of preparing an all-organic actuator (cross-section view) and (b) a prototype actuator film.

Kang, Park, Lowther, Harrison, and Park 


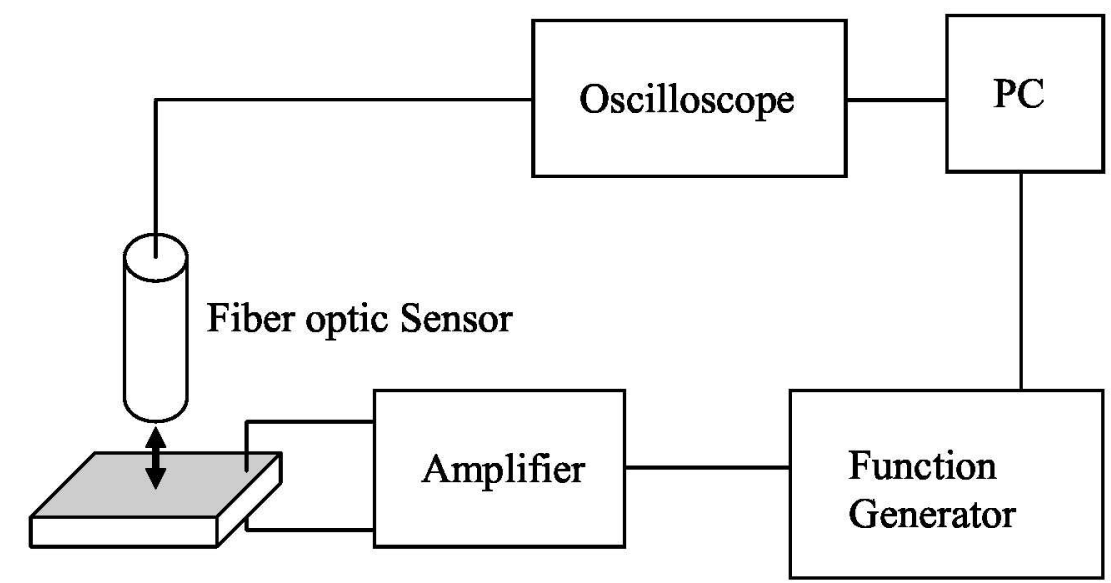

Actuation Sample

Figure 4. Schematic diagram of apparatus used to measure electromechanical actuation.

Kang, Park, Lowther, Harrison, and Park 
(a)

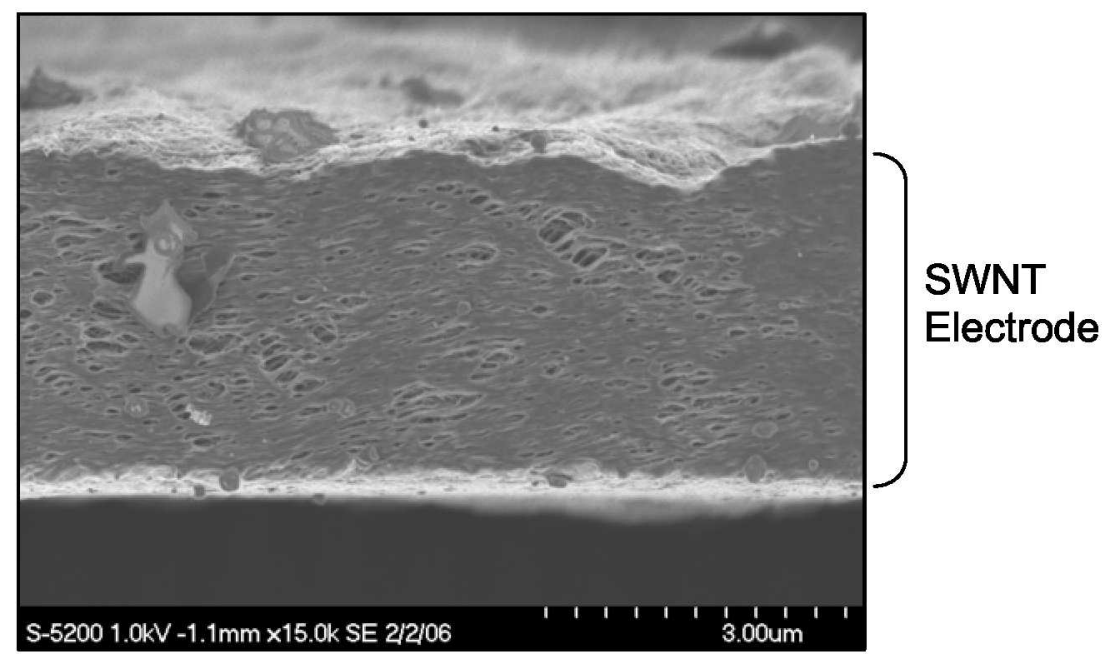

(b)

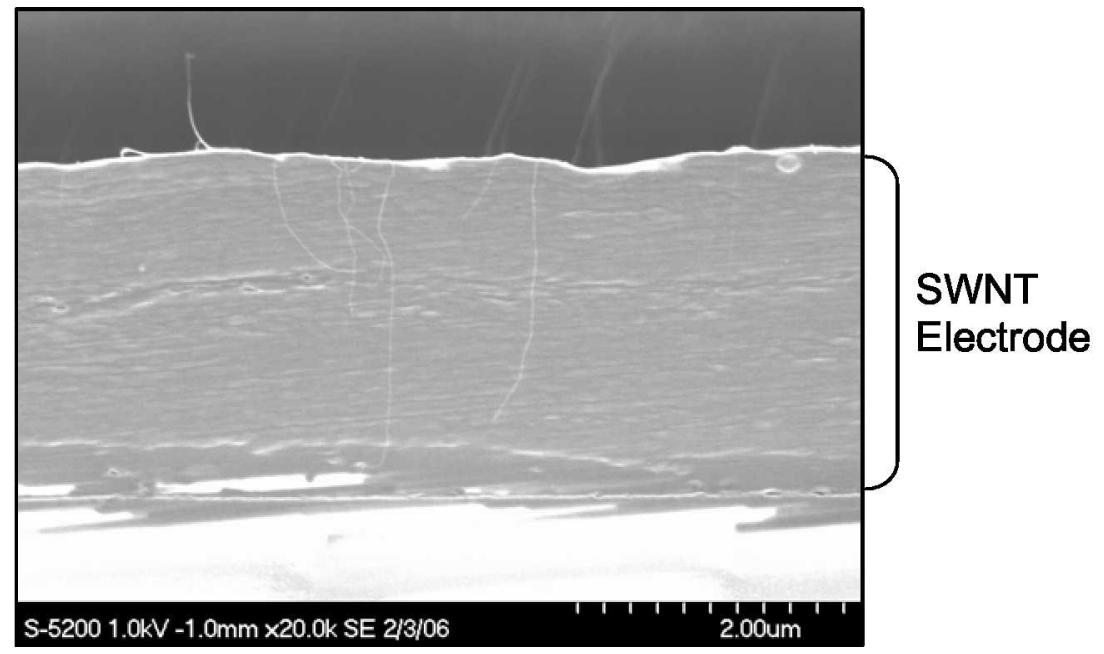

Figure 5. The cross-sectional SEM images of SWNT electrode (a) after pressing at 600 psi and (b) after pressing at 6000 psi.

Kang, Park, Lowther, Harrison, and Park 
(a)

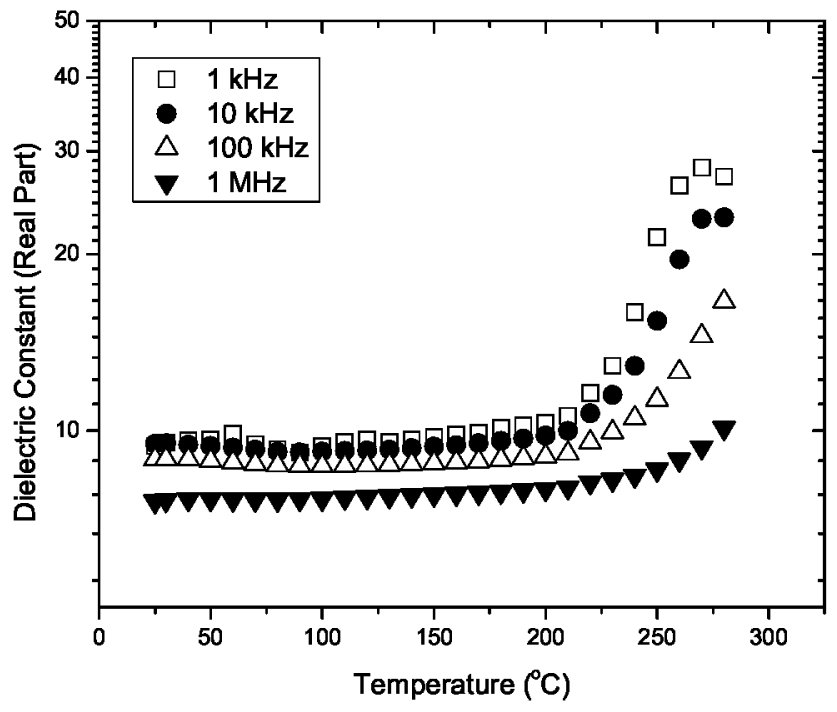

(b)

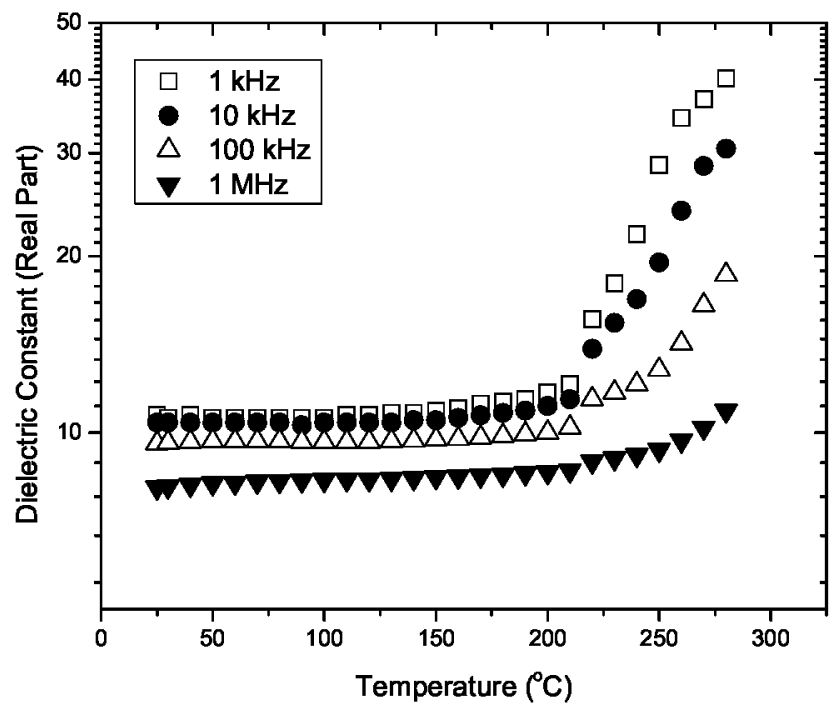

Figure 6. The dielectric constant of (a) SWCNT/LaRC-EAP nanocomposite with SWNT electrodes and (b) SWCNT/LaRC-EAP nanocomposite with metal electrodes as a function of temperature and frequency.

Kang, Park, Lowther, Harrison, and Park 


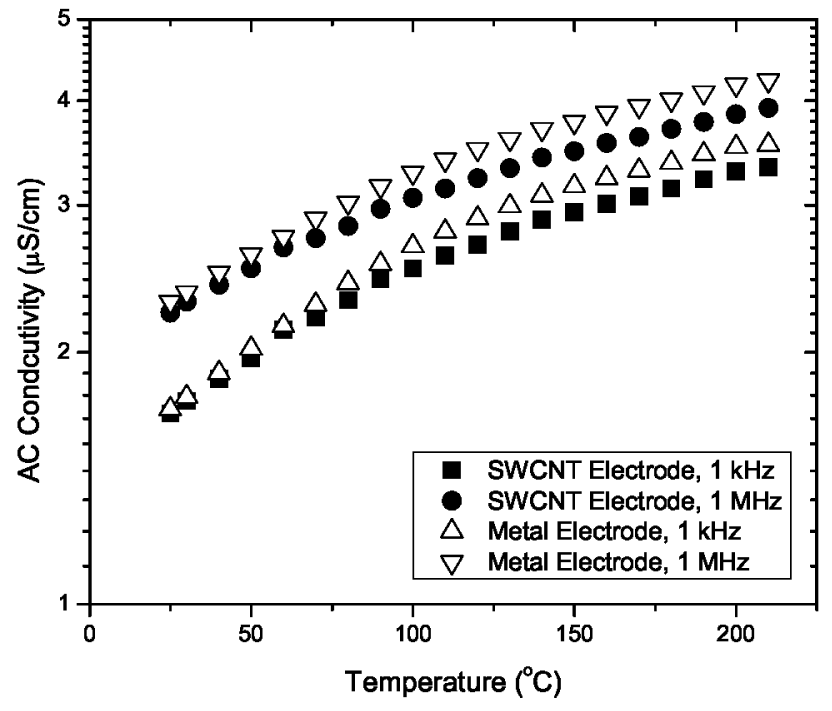

Figure 7. AC conductivities of SWCNT/LaRC-EAP nanocomposite layered with SWNT electrodes $(\square)$ and layered with metal electrodes $(\Delta \nabla)$ as a function of temperature and frequency.

Kang, Park, Lowther, Harrison, and Park 


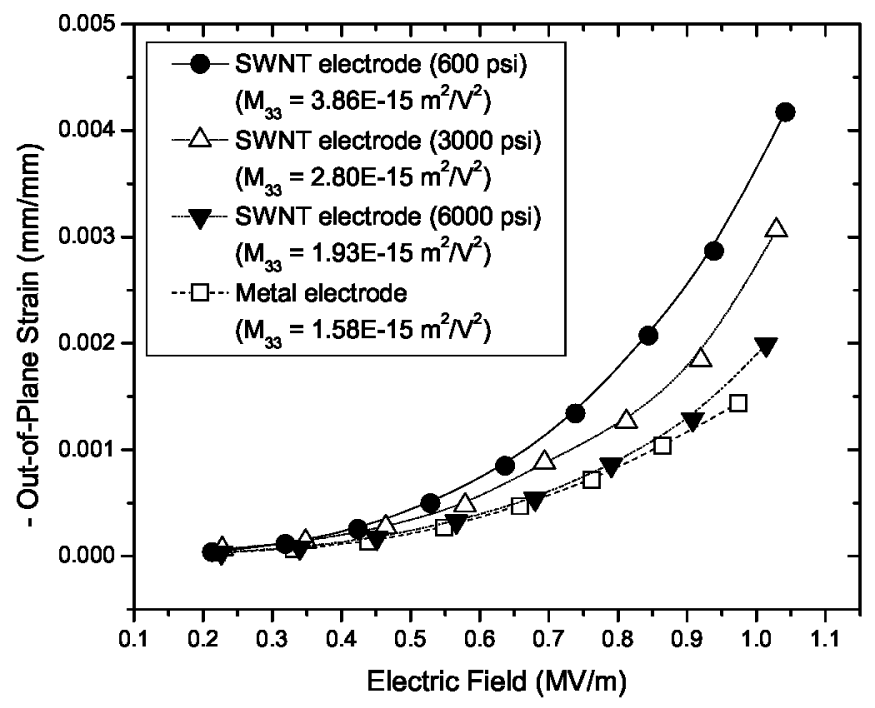

Figure 8. The electric field-induced strain of SWCNT/LaRC-EAP nanocomposite layered with metal electrodes $(\square)$ and with SWNT electrodes $(\triangle \triangle \nabla)$.

Kang, Park, Lowther, Harrison, and Park 